\title{
$\beta$-Blockers are associated with a reduction in COPD exacerbations
}

\author{
Surya P Bhatt, ${ }^{1}$ James M Wells, ${ }_{1}{ }^{1}$ Gregory L Kinney, ${ }^{2}$ George R Washko Jr, ${ }^{3}$ \\ Matthew Budoff, ${ }^{4}$ Young-il Kim, ${ }^{5}$ William C Bailey, ${ }_{1}^{1}$ Hrudaya Nath, ${ }^{6}$ \\ John E Hokanson, ${ }^{2}$ Edwin K Silverman, ${ }^{7}$ James Crapo, ${ }^{8}$ Mark T Dransfield, ${ }^{1,9}$ \\ For the COPDGene Investigators
}

\begin{abstract}
- Additional material is published online only. To view please visit the journal online (http://dx.doi.org/10.1136/ thoraxjnl-2015-207251)
\end{abstract}

For numbered affiliations see end of article.

\section{Correspondence to} Dr Surya P Bhatt, University of Alabama at Birmingham, Division of Pulmonary, Allergy and Critical Care Medicine, THT 422, 1720, 2nd Avenue South, Birmingham, AL 35294, USA; spbhatt@uab.edu

Received 30 April 2015 Revised 29 June 2015 Accepted 21 July 2015 Published Online First 17 August 2015

\section{CrossMark}

To cite: Bhatt SP, Wells JM, Kinney GL, et al. Thorax 2016;71:8-14.

\begin{abstract}
Background While some retrospective studies have suggested that $\beta$-blocker use in patients with COPD is associated with a reduction in the frequency of acute exacerbations and lower mortality, there is concern that their use in patients with severe COPD on home oxygen may be harmful.

Methods Subjects with Global Initiative for Chronic Obstructive Lung Disease (GOLD) stage 2-4 COPD participating in a prospective follow-up of the COPDGene cohort, a multicentre observational cohort of current and former smokers were recruited. Total and severe exacerbation rates were compared between groups categorised by $\beta$-blocker use on longitudinal follow-up using negative binomial regression analyses, after adjustment for demographics, airflow obstruction, \%emphysema on $\mathrm{CT}$, respiratory medications, presence of coronary artery disease, congestive heart failure and coronary artery calcification, and after adjustment for propensity to prescribe $\beta$-blockers.
\end{abstract}

Results 3464 subjects were included. During a median of 2.1 years of follow-up, $\beta$-blocker use was associated with a significantly lower rate of total (incidence risk ratio (IRR) $0.73,95 \% \mathrm{Cl} 0.60$ to $0.90 ; p=0.003$ ) and severe exacerbations (IRR $0.67,95 \% \mathrm{Cl} 0.48$ to 0.93 ; $\mathrm{p}=0.016)$. In those with GOLD stage 3 and 4 and on home oxygen, use of $\beta$-blockers was again associated with a reduction in the rate of total (IRR $0.33,95 \% \mathrm{Cl}$ 0.19 to $0.58 ; p<0.001$ ) and severe exacerbations (IRR $0.35,95 \% \mathrm{Cl} 0.16$ to $0.76 ; p=0.008)$. Exacerbation reduction was greatest in GOLD stage $B$. There was no difference in all-cause mortality with $\beta$-blocker use.

Conclusions $\beta$-Blockers are associated with a significant reduction in COPD exacerbations regardless of severity of airflow obstruction. The findings of this study should be tested in a randomised, placebo-controlled trial.

Trial registration number (ClinicalTrials.gov NCT00608764).

\section{BACKGROUND}

COPD is now the third leading cause of death in the USA, and the majority of COPD-related morbidity and healthcare costs is due to acute exacerbations. ${ }^{1}$ Unfortunately, current therapies targeting airflow limitation and pulmonary inflammation only result in a modest reduction in exacerbation risk even when treatments are used in

\section{Key messages}

What is the key question?

- What is the impact of $\beta$-blockers on the rate of exacerbations in COPD?

What is the bottom line?

- We show that $\beta$-blockers are associated with a significant reduction in COPD exacerbations, an effect not seen with other cardiac medications, though this potential benefit should be tested in a randomised trial.

\section{Why read on?}

- The data also suggest that $\beta$-blockers are safe and associated with reduced exacerbations in subjects with severe COPD and on home oxygen, a subgroup that was considered at especially high risk for complications from $\beta$-blocker use.

combination. ${ }^{2}$ There is increasing recognition that COPD is a multisystem disease associated with a number of significant comorbid illnesses including diagnosed and subclinical cardiovascular disease, which can cause or contribute to moderate exacerbations managed in the outpatient setting as well as more severe events requiring hospitalisation. ${ }^{3}$

Despite concerns that $\beta$-blockers may worsen lung function in patients with COPD, ${ }^{45}$ randomised trials do not support this fear ${ }^{6}$ and multiple retrospective studies have in fact suggested that they are associated with a reduction in overall mortality, ${ }^{7-12}$ a reduction in the frequency of acute exacerbations ${ }^{10}{ }^{12-14}$ and improved survival when continued in-hospital during an exacerbation. ${ }^{15}{ }^{16}$ This may be due to cardioprotective effects, ${ }^{17}$ though pulmonary-specific benefits could also play a role. These findings are in sharp contrast to those from the single prospective study examining the issue, which reported that $\beta$-blocker use in patients with severe COPD on home oxygen is associated with increased mortality. ${ }^{18}$ We sought to revisit this question by analysing prospectively collected data from the well-characterised COPDGene cohort ${ }^{19}$ and hypothesised that $\beta$-blockers would be associated with a reduction in exacerbations. We also analysed the relationships between calcium channel 
blockers (CCBs) and ACE inhibitors (ACEIs)/angiotensin receptor blockers (ARBs) and the rate of exacerbations.

\section{METHODS}

\section{Study population and assessments}

Subjects from a large multicentre cohort study (COPDGene) with Global Initiative for Chronic Obstructive Lung Disease (GOLD) stage 2-4 were included in the study. ${ }^{1}$ The COPDGene study enrolled a total of 10300 persons, of whom 3464 participants had stage 2-4 COPD. The COPDGene study details have been previously published. ${ }^{19}$ Briefly, current and former smokers aged 45-80 years were enrolled, and those with known lung disease other than COPD and asthma were excluded. Diagnosis of COPD was made using the post-bronchodilator ratio of $\mathrm{FEV}_{1}$ to the $\mathrm{FVC}$ of $<0.70 .{ }^{1}$ Respiratory morbidity was assessed at baseline, and this included the 6 min walk distance, respiratory disease-related health impairment and quality of life (St George's Respiratory Questionnaire (SGRQ) score), and dyspnoea using the Modified Medical Research Council (MMRC) dyspnoea score. ${ }^{19}$ Subjects were categorised into GOLD stages A-D per GOLD COPD guidelines. ${ }^{1}$ Since we did not measure COPD Assessment Test scores, we used SGRQ as a substitute to derive GOLD stages. ${ }^{20}$

High-resolution CT scans were performed at full inspiration and after exhalation. Emphysema was estimated by the percentage of lung volume on the inspiratory CT with attenuation $<-950$ Hounsfield Units (HU) (low attenuation area, \%LAA950insp), and gas trapping by the percentage of lung volume on the expiratory CT with attenuation $<-856 \mathrm{HU}\left(\% \mathrm{LAA} 856_{\text {exp }}\right.$ ), using 3D Slicer software (http://www.airwayinspector.org). ${ }^{19}$ Airway wall thickness was measured by wall area percentage at the segmental and subsegmental level using Pulmonary Workstation 2 (VIDA Diagnostics, Coralville, Iowa, USA).$^{19}$ Coronary artery calcification (CAC) was measured using Agatston scores. ${ }^{21}$ In order to examine relationship between $\beta$-blocker use and significant coronary artery disease (CAD), we used Agatston score $>400$ to indicate presence of significant CAD.

Comorbidities were recorded as patient-reported physiciandiagnosed conditions. Data on medication use were obtained at the time of enrolment as reported by participants, and when available, by inspecting their medication containers. Medication history included use of respiratory inhalers such as short-acting and long-acting $\beta$ agonists, short-acting and long-acting anticholinergics, and inhaled corticosteroids. Relevant cardiac medications including $\beta$-blockers, CCBs, ACEIs and ARBs were also recorded.

Exacerbations were defined as worsening of respiratory symptoms requiring use of either antibiotics or systemic steroids, and those requiring hospitalisation were termed severe exacerbations. ${ }^{1}$ Data on vital status were also obtained.

\section{Statistical analyses}

Subjects were categorised by use of three major categories of cardiac medications ( $\beta$-blockers, CCBs and combined ACEI/ ARBs). Bivariate comparisons were made between those using and not using these drugs using $\chi^{2}$ test for categorical variables such as race, gender, $\mathrm{CAD}$, congestive heart failure (CHF) and the use of long-acting respiratory medications (long-acting $\beta$-agonists/inhaled corticosteroids and long-acting antimuscarinics), and two-tailed two sample t tests for continuous variables including age, pack-years of smoking, body mass index (BMI), $\mathrm{FEV}_{1}$, \%emphysema on CT and log CAC. Data for CAC were $\log$ transformed due to its non-normal distribution.
Due to over-dispersion of exacerbations, negative binomial regression was performed for the primary analyses of rates of exacerbation on follow-up in patients taking $\beta$-blockers compared with those who were not. Predictors of exacerbations were selected based on previous COPD studies examining this issue, ${ }^{22} 23$ and $\log$ CAC was included as a potentially new predictor. ${ }^{24}$ Variables significant on bivariate analyses at a two-sided significance level of 0.05 were included in the multivariable negative binomial model. To identify potential multicollinearity problems, variance inflation factor (VIF) was estimated. Variables with VIF $>5$ were excluded from the model. Similar analyses were repeated for CCBs and ACEI/ARBs. To assess the robustness of the negative binomial models, we also compared the time to first contact at which an exacerbation was captured by the longitudinal follow-up system using Cox proportional hazards models. Unadjusted comparisons were first made for each covariate with time to exacerbation reporting as the outcome variable and those variables significant with a $\mathrm{p}$ value $<0.05$ were included to adjust HR. The aforementioned analyses were repeated for CCBs and ACEI/ARBs. Proportionality assumptions were tested by using log-log plot for categorical variables and Schoenfeld residual plots for each of the predictors.

\section{Propensity score analysis}

As $\beta$-blockers are more likely to be prescribed to patients with $\mathrm{CAD}$ and $\mathrm{CHF}$, and there is a tendency to underprescribe the drugs in those with COPD, propensity score was used to adjust for covariates associated with $\beta$-blocker use. ${ }^{25} \mathrm{~A}$ logistic regression model was first created with $\beta$-blocker use as the dependent variable and demographics including age, race, gender, BMI, smoking history in pack-years, CAD, CHF and COPD severity by GOLD stage (2-4) as covariates. The predicted probability of treatment with $\beta$-blocker was calculated from this regression model and was the propensity score used in an effort to balance the distribution of measured baseline covariates between treated and non-treated groups. The propensity score as a continuous variable was then entered into the original regression model to compare rates of exacerbations in those who were and were not receiving $\beta$-blockers. Sensitivity analyses were performed by adding baseline dyspnoea and quality of life as measured by MMRC and SGRQ scores to the propensity scores.

To assess survival with $\beta$-blocker use, Cox proportional hazards analysis was performed. Single predictor models were evaluated for demographics including age, race, gender, BMI, pack-years, CAD, CHF, long-acting respiratory medications, $\mathrm{FEV}_{1}$, \%emphysema on CT and log CAC, respectively. Those variables significant at a two-sided $\alpha$ level of 0.05 were included in the multivariable model to assess adjusted HR for mortality with $\beta$-blocker use. Final results were determined to be statistically significant when the accompanying statistical test yielded a two-tailed probability of $\leq 0.05$. All analyses were performed using Statistical Package for the Social Sciences (SPSS V.22.0, SPSS, Chicago, Illinois, USA).

\section{RESULTS}

\section{Subject characteristics}

In total, 3464 subjects were included with a mean age (SD) of $63.4 \pm 8.5$ years. Females comprised $44.4 \%(n=1537)$ of the cohort, 765 (22.1\%) were African-American and 1410 (40.7\%) were active smokers. Patients spanned the spectrum of COPD disease severity with 1831 (52.9\%), $1092(31.5 \%)$ and 541 (15.6\%) having GOLD stage 2, 3 and 4, respectively. The mean per cent predicted $\mathrm{FEV}_{1}$ was $50.3 \pm 17.8,955$ (27.6\%) were on 
home oxygen therapy and $777(22.4 \%)$ subjects had a severe exacerbation in the year prior to enrolment. CAD was present in $565(16.3 \%)$ of patients of whom $212(37.5 \%)$ were on $\beta$-blockers. CHF was present in $181(5.2 \%)$ of whom $58(32.0 \%)$ were on $\beta$-blockers. More than half reported a history of hypertension.

Table 1 shows a comparison of the baseline characteristics and comorbidities for those receiving $\beta$-blockers versus those not prescribed these drugs. Similar comparisons for CCBs and ACE/ARBs are provided in online supplementary table S1. When compared with those not on $\beta$-blockers, those receiving these medications were older, had a greater smoking burden and had a higher BMI. African-Americans were significantly less likely to be taking $\beta$-blockers compared with Caucasians. Resting heart rate was lower in those on $\beta$-blockers compared with those not on the medication $(69.5 \pm 12.0$ vs $78.8 \pm 12.8$; $\mathrm{p}<0.001)$. The overall propensity score for those on $\beta$-blockers was higher than in those not on $\beta$-blockers $(0.25 \pm 0.18$ vs 0.12 $\pm 0.11 ; \mathrm{p}<0.001)$. Addition of MMRC and SGRQ to the propensity scores resulted in persistent differences but no significant

Table 1 Baseline characteristics of subjects on $\beta$-blockers

\begin{tabular}{|c|c|c|c|}
\hline & \multicolumn{3}{|l|}{ 及-blocker } \\
\hline & Yes $(n=474)$ & No $(n=2990)$ & $p$ Value \\
\hline Age (years) & $66.8(7.7)$ & $62.8(8.5)$ & $<0.001$ \\
\hline Gender, female, \% & 189 (39.9) & $1348(45.1)$ & 0.034 \\
\hline Race, African-American, \% & $73(15.4)$ & $692(23.1)$ & $<0.001$ \\
\hline BMI $\left(\mathrm{kg} / \mathrm{m}^{2}\right)$ & $29.9(6.7)$ & $27.8(6.2)$ & $<0.001$ \\
\hline Pack-years of smoking & $56.8(30.0)$ & $52.6(27.0)$ & 0.002 \\
\hline Heart rate (per minute) & $69.5(12.0)$ & $78.8(12.8)$ & $<0.001$ \\
\hline $\mathrm{FEV}_{1}(\mathrm{~L})$ & $1.50(0.54)$ & $1.44(0.64)$ & 0.036 \\
\hline $\mathrm{FEV}_{1} \%$ predicted & $53.2(15.4)$ & $49.8(18.2)$ & $<0.001$ \\
\hline $\mathrm{FEV}_{1} / \mathrm{FVC}$ & $0.53(0.11)$ & $0.49(0.13)$ & $<0.001$ \\
\hline GOLD stage, $\%$ & & & $<0.001$ \\
\hline 2 & $292(61.6)$ & $1539(51.5)$ & \\
\hline 3 & $144(30.4)$ & 948 (31.7) & \\
\hline 4 & $38(8.0)$ & $503(16.8)$ & \\
\hline Home oxygen therapy, \% & $100(21.1)$ & 855 (28.6) & 0.001 \\
\hline Severe exacerbation in prior year, \% & 92 (19.4) & $685(22.9)$ & 0.090 \\
\hline Asthma, \% & 99 (20.9) & $830(27.8)$ & 0.002 \\
\hline Coronary artery disease, $\%$ & $212(44.7)$ & $353(11.8)$ & $<0.001$ \\
\hline Peripheral vascular disease, \% & $37(7.8)$ & $83(2.8)$ & $<0.001$ \\
\hline Cerebrovascular disease, \% & $51(10.8)$ & $155(5.2)$ & $<0.001$ \\
\hline Congestive heart failure, \% & $58(12.2)$ & $123(4.1)$ & $<0.001$ \\
\hline Hypertension, \% & $400(84.4)$ & $1356(45.4)$ & $<0.001$ \\
\hline Diabetes mellitus, \% & $114(24.1)$ & $326(10.9)$ & $<0.001$ \\
\hline Emphysema (\%LAA<950 insp) & $9.9(10.5)$ & $13.4(12.9)$ & $<0.001$ \\
\hline Gas trapping $\left(\%\right.$ LAA $<856_{\text {exp }}$ ) & $34.8(18.8)$ & 39.7 (20.9) & $<0.001$ \\
\hline Wall area \% segmental & $62.8(3.0)$ & $62.7(3.0)$ & 0.567 \\
\hline CAC & $432.6(573.9)$ & $212.2(397.5)$ & $<0.001$ \\
\hline MMRC & $2.0(1.4)$ & $2.1(1.4)$ & 0.079 \\
\hline SGRQ & $36.6(20.2)$ & $41.3(22.1)$ & $<0.001$ \\
\hline 6MWD (m) & 349 (127) & 351 (133) & 0.776 \\
\hline BODE & $2.7(1.9)^{\star *}$ & $3.0(2.1)$ & 0.004 \\
\hline
\end{tabular}

\%LAA $<856_{\text {exp }}$ \%low attenuation area below a threshold of -856 Hounsfield Units at end tidal expiration; \%LAA $<950_{\text {insp }} \%$ low attenuation area below a threshold of -950 Hounsfield Units at end inspiration; 6MWD, 6 min walk distance; BMI, body mass index; $B O D E$, body mass index, airflow obstruction, dyspnoea and exercise capacity index; CAC, coronary artery calcification as measured by Agatston score; GOLD, Global Initiative for Chronic Obstructive Lung Disease; MMRC, Modified Medical Research Council dyspnoea scale; SGRQ, St. George's Respiratory Questionnaire; Wall area\%, bronchial wall area at segmental level. increment in probability $(0.26 \pm 0.18$ vs $0.12 \pm 0.11 ; \mathrm{p}<0.001)$ for those on and not on $\beta$-blockers, respectively. $\beta$-blocker use declined with increasing disease severity as assessed by GOLD stage and by the extent of CT emphysema and was less common in subjects on home oxygen therapy. As expected, those on $\beta$-blockers were more likely to have $\mathrm{CHF}$ and $\mathrm{CAD}$, but were less likely to have asthma. They also had better respiratory quality of life (table 1) and were less often treated with both maintenance and short-acting rescue respiratory medications (table 2). Cardiovascular disease was also more common in patients taking CCBs and ACEI/ARBs (see online supplementary table S1). However, in contrast to $\beta$-blockers, there was no difference in respiratory quality of life or in the severity of disease by GOLD stage between patients who were taking and not taking $\mathrm{CCBs}$ and $\mathrm{ACEI} / \mathrm{ARBs}$.

\section{Exacerbations}

We had follow-up data on 2919 subjects (median 2.1 years, range 0.2 to 4.2 years). Figure 1 shows the adjusted incidence risk ratios (IRR) for the number of total and severe exacerbations occurring during long-term follow-up. Use of $\beta$-blockers was associated with a significantly lower rate of total (IRR 0.73 , 95\% CI 0.60 to $0.90 ; p=0.003$ ) and severe exacerbations (IRR $0.67,95 \%$ CI 0.48 to $0.93 ; \mathrm{p}=0.016$ ). After adjustment for the propensity to prescribe $\beta$-blockers due to the presence of CAD, CHF and COPD disease severity by GOLD stage, $\beta$-blocker use was again associated with a reduction in total exacerbations (IRR $0.71,95 \%$ CI 0.58 to $0.87 ; \mathrm{p}=0.001$ ) and also severe exacerbations (IRR 0.59 , 95\% CI 0.42 to $0.83 ; \mathrm{p}=0.002$ ) (figure 1). When baseline dyspnoea as measured by MMRC and respiratory quality of life (SGRQ) were added to the propensity model, the impact of $\beta$-blockers on exacerbation rate was unchanged (IRR $0.75,95 \%$ CI 0.61 to $0.93 ; p=0.008$ and IRR $0.63,95 \%$ CI 0.45 to $0.89 ; p=0.009$ for total and severe exacerbations, respectively) (figure 1).

Based on the previous report of Ekstrom et al suggesting that $\beta$-blockers could be harmful in patients with severe and very severe disease, we also separately analysed the relationship between $\beta$-blockers and exacerbations in patients with GOLD stage 3 and 4 and on home oxygen therapy $(n=743)$. Of these patients, $152(20.5 \%)$ had CAD and $27(17.8 \%)$ of them were on $\beta$-blockers. The mean age of this group was $65.5 \pm 7.8$ years and mean $\mathrm{FEV}_{1} \%$ predicted was 29.8 9 9.9. After adjustment, use of $\beta$-blockers was again associated with a significant

Table 2 Comparison of respiratory medications

\begin{tabular}{|c|c|c|c|}
\hline & \multicolumn{3}{|l|}{ 及-Blocker } \\
\hline & Yes $(n=474)$ & No $(n=2990)$ & p Value \\
\hline SABA, $\%$ & $264(56.8)$ & $1791(60.7)$ & 0.105 \\
\hline SAMA, $\%$ & $65(14.3)$ & $598(20.6)$ & 0.002 \\
\hline $\mathrm{LABA}^{*}, \%$ & $188(39.7)$ & $1433(47.9)$ & 0.001 \\
\hline LAMA, \% & $174(37.6)$ & $1166(40.1)$ & 0.297 \\
\hline ICS* $\%$ & $191(40.3)$ & $1494(50.0)$ & $<0.001$ \\
\hline Combination ICS/LABA, \% & $173(37.9)$ & $1324(46.2)$ & 0.001 \\
\hline Triple therapy, \% & $102(22.4)$ & $814(28.4)$ & 0.008 \\
\hline Theophylline, \% & $9(2.3)$ & $138(4.7)$ & 0.027 \\
\hline Systemic steroids, \% & $11(2.8)$ & $178(6.1)$ & 0.008 \\
\hline
\end{tabular}

* This medication was used alone or in combination.

ICS, inhaled corticosteroid; LABA, long-acting $\beta$-agonist; LAMA, long-acting

muscarinic antagonist; SABA, short-acting $\beta$-agonist; SAMA, short-acting muscarinic antagonist; Triple therapy, ICS+LABA+LAMA. 
Incidence Risk Ratio for Total Exacerbations

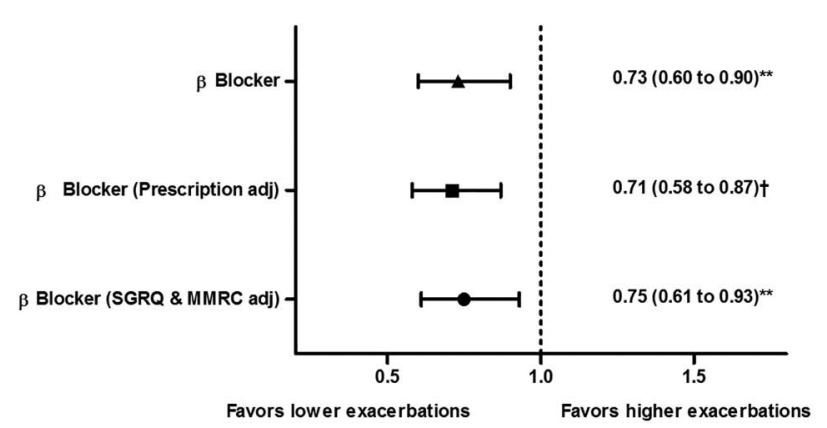

Incidence Risk Ratio for Severe Exacerbations

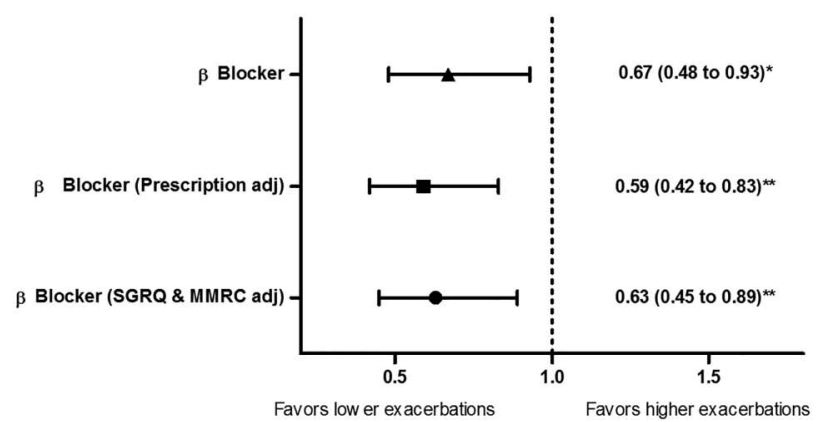

Figure 1 The comparison of adjusted incidence risk ratios (IRRs) for total and severe exacerbations occurring during long-term follow-up in patients with COPD who are on or not on $\beta$-blocker therapy. These rates were adjusted for total exacerbations by age, gender, race, smoking burden, body mass index, FEV1, \%emphysema on CT, coronary artery calcification (CAC), presence of congestive heart failure and coronary artery disease, long-acting respiratory medications and for the propensity to prescribe $\beta$-blockers based on demographics, coronary artery disease, congestive heart failure and severity of airflow obstruction. Rates were adjusted for severe exacerbations by age, race, FEV1, \%emphysema on CT, $\log C A C$, presence of congestive heart failure, long-acting respiratory medications and for the propensity to prescribe $\beta$-blockers. All values expressed as IRRs (95\% Cls). SGRQ, St. George's Respiratory Questionnaire; MMRC, Modified Medical Research Council dyspnoea scale; Prescription adj, adjusted for propensity to prescribe $\beta$-blockers based on demographics, coronary artery disease, congestive heart failure and severity of airflow obstruction; SGRQ and MMRC adj, adjusted for propensity to prescribe $\beta$-blockers based on demographics, coronary artery disease, congestive heart failure and severity of airflow obstruction, as well as respiratory quality of life using SGRQ and dyspnoea per MMRC $s c o r e .{ }^{* *} p<0.01 ;+p<0.001$.

reduction in the rate of total (IRR $0.33,95 \%$ CI 0.19 to 0.58 ; $\mathrm{p}<0.001$ ) and severe exacerbations (IRR $0.35,95 \%$ CI 0.16 to $0.76 ; \mathrm{p}=0.008)$. On propensity adjustment using the above factors as well as baseline MMRC and SGRQ, the exacerbation rate remained lower with the use of $\beta$-blockers (IRR for total exacerbations $0.34,95 \%$ CI 0.19 to $0.60 ; \mathrm{p}<0.001$, and IRR for severe exacerbations $0.36,95 \%$ CI 0.17 to $0.79 ; \mathrm{p}=0.010$ ). We also specifically examined the relationship between nonselective $\beta$-blockers and exacerbation rates. Only $52(2.1 \%)$ were on non-selective $\beta$-blockers and no differences in exacerbations with use of these medications were observed (adjusted IRR for total and severe exacerbations $1.16,95 \%$ CI 0.72 to 1.87 ;

Table 3 Comparison of adjusted incidence risk ratios (IRR) for total and severe exacerbations with $\beta$-blocker therapy across GOLD stages A-D

\begin{tabular}{|c|c|c|c|c|}
\hline & IRR & \multicolumn{2}{|c|}{$95 \% \mathrm{Cl}$} & p Value \\
\hline \multicolumn{5}{|c|}{$A(n=719)$} \\
\hline Total & 2.02 & 1.20 & 3.38 & 0.008 \\
\hline Severe & 0.91 & 0.31 & 2.65 & 0.862 \\
\hline \multicolumn{5}{|c|}{$B(n=856)$} \\
\hline Total & 0.45 & 0.28 & 0.74 & 0.002 \\
\hline Severe & 0.27 & 0.09 & 0.76 & 0.014 \\
\hline \multicolumn{5}{|c|}{$C(n=201)$} \\
\hline Total & 1.27 & 0.49 & 3.26 & 0.626 \\
\hline Severe & 0.50 & 0.04 & 6.06 & 0.590 \\
\hline \multicolumn{5}{|c|}{$D(n=1688)$} \\
\hline Total & 0.68 & 0.51 & 0.92 & 0.011 \\
\hline Severe & 0.71 & 0.47 & 1.06 & 0.097 \\
\hline \multicolumn{5}{|c|}{$\begin{array}{l}\text { Total exacerbation rates adjusted for age, gender, } \mathrm{FEV}_{1}, \% \text { emphysema on } \mathrm{CT} \text {, } \\
\text { coronary artery calcification, presence of CAD, long-acting respiratory medications and } \\
\text { for the propensity to prescribe } \beta \text {-blockers based on demographics, CAD, congestive } \\
\text { heart failure and severity of airflow obstruction. } \\
\text { Severe exacerbation rates adjusted for age, race, FEV1, \%emphysema on CT, coronary } \\
\text { artery calcification, presence of congestive heart failure, long-acting respiratory } \\
\text { medication, and for the propensity to prescribe } \beta \text {-blockers. All values expressed as } \\
\text { IRRs (95\% CIs). } \\
\text { GOLD, Global Initiative for Chronic Obstructive Lung Disease. }\end{array}$} \\
\hline
\end{tabular}

$\mathrm{p}=0.546$, and $1.12,95 \%$ CI 0.51 to $2.44 ; \mathrm{p}=0.774$, respectively).

We further analysed the association between exacerbations and the use of $\beta$-blockers by the new GOLD stage scheme (table 3). There were 719, 856, 201 and 1688 subjects in GOLD stage A-D, and CAD was present in $12.9 \%, 16.5 \%, 10.0 \%$ and $18.4 \%$, respectively. In adjusted analyses, $\beta$-blocker use was associated with the greatest reduction in exacerbation risk in GOLD stage B though an increased risk was observed in stage A patients (for total but not severe events).

We also examined the relationship between $\beta$-blocker use and exacerbation rates in subjects with and without significant CAC (Agatston score >400). Of the 3464 subjects, $620(18 \%)$ had significant CAC. When compared with those with Agatston score $<400$, those with score $>400$ were more likely to have CAD $(31.1 \%$ vs $12.9 \% ; \mathrm{p}<0.001)$ and $\mathrm{CHF}(7.3 \%$ vs $4.8 \%$; $\mathrm{p}=0.01)$, and were more likely to be on $\beta$-blockers $(24.7 \%$ vs $11.2 \% ; \mathrm{p}<0.001)$. In those with significant CAC, $\beta$-blockers were associated with a significant reduction in total (IRR 0.67, 95\% CI 0.48 to $0.93 ; \mathrm{p}=0.019)$ but not severe exacerbations (IRR 0.90, 95\% CI 0.54 to $1.50 ; \mathrm{p}=0.692$ ). In those with CAC $<400, \beta$-blockers were associated with a significant reduction in both total and severe exacerbations (IRR 0.76 , 95\% CI 0.58 to $0.99 ; \mathrm{p}=0.045$ and $0.55,95 \%$ CI 0.34 to $0.86 ; \mathrm{p}=0.010$, respectively).

In contrast, the use of CCBs in the cohort was associated with a significantly increased risk of severe exacerbations (IRR $1.65,95 \%$ CI 1.23 to $2.22 ; \mathrm{p}=0.001)$. The rate of severe exacerbations was not related to the use of ACEI/ARBs and neither ACEI/ARBs nor CCBs were associated with the rate of total exacerbations (see online supplementary figure S1).

\section{Time to first exacerbation}

The use of $\beta$-blockers was associated with a longer time to first severe exacerbation (unadjusted HR $075,95 \%$ CI 0.57 to 0.99 ; $\mathrm{p}=0.048$ and after adjustment for age, race, $\mathrm{CHF}, \mathrm{FEV}_{1}, \%$ emphysema on CT, respiratory medications, log CAC and the propensity to prescribe $\beta$-blockers, HR 0.69 , 95\% CI 0.47 to $1.02 ; p=0.060$ ) (see online supplemental figure S2). $\beta$-Blocker use did not impact time to first total exacerbation (adjusted HR 
$0.91,95 \%$ CI 0.75 to $1.11 ; \mathrm{p}=0.363$ ) (see online supplemental figure S2). There was no difference in time to first event with CCBs (adjusted HR for total $=1.05$, 95\% CI 0.83 to 1.32; $\mathrm{p}=0.697$ and severe $=1.05,95 \%$ CI 0.75 to $1.47 ; \mathrm{p}=0.774$ ), and with ACEI/ARBs (adjusted HR for total=1.01, 95\% CI 0.84 to $1.21 ; \mathrm{p}=0.950$ and severe $=1.07,95 \%$ CI 0.82 to 1.41 ; $\mathrm{p}=0.615)$

\section{Mortality}

In the entire cohort, 242 (7\%) patients died over the period of follow-up. After adjustment for demographics including age, race, gender, smoking burden in pack-years and BMI, CAD, $\mathrm{CHF}, \log \mathrm{CAC}, \mathrm{FEV}_{1}$, \%emphysema on CT and respiratory medications, use of $\beta$-blockers was not associated with mortality in the entire population (adjusted HR 0.85 , 95\% CI 0.54 to $1.32 ; \mathrm{p}=0.47)$ or in those with GOLD stage 3 and 4 and on home oxygen, of whom 119 (18.6\%) died (HR for $\beta$-blockers $0.71,95 \%$ CI 0.32 to $1.55 ; \mathrm{p}=0.39$ ). There was also no difference in mortality with use of CCBs (HR $0.78,95 \%$ CI 0.49 to $1.25 ; \mathrm{p}=0.30$ ) and ACEI/ARBs (HR $0.97,95 \%$ CI 0.67 to $1.41 ; \mathrm{p}=0.88)$ in the entire cohort.

\section{DISCUSSION}

We found that the use of $\beta$-blockers by subjects with moderate to very severe COPD is associated with a significant reduction in exacerbations and that this benefit persists after adjustment for underlying cardiovascular disease as well as the propensity to receive the drugs. We also found that the use of other cardiac medications such as CCBs and ACEI/ARBs was not associated with a reduction in exacerbation risk, suggesting that the reduction seen with $\beta$-blockers is a class effect. We add to existing literature by suggesting beneficial effects of $\beta$-blockers in COPD using prospectively collected data from a well-characterised cohort with measures of respiratory morbidity, spirometrically proven COPD and CT measures of emphysema and CAC.

Because of the concern that $\beta$-blockers may trigger bronchoconstriction and worsening of lung function, they are significantly underprescribed in patients with COPD even in those with established cardiovascular disease in whom the drugs have known mortality and morbidity benefits. ${ }^{5}$ This practice pattern persists and was observed in our population despite multiple studies showing that there is no significant detrimental effect on lung function, especially with cardioselective $\beta$-blockers, even in patients with severe airflow limitation. ${ }^{6}$ There is also now clear evidence that COPD is associated with accelerated atherosclerosis and a greater frequency of subclinical CAD and cardiac dysfunction $^{26}$ and thus $\beta$-blockers may also have benefits in patients without diagnosed cardiovascular disease. This is supported by our finding that the drugs reduced exacerbation rates regardless of the severity of coronary calcification. Cardiovascular risk is particularly accentuated during acute exacerbations that are associated with elevated levels of prognostic markers of cardiac injury and stretch (troponin and $\mathrm{N}$-terminal brain natriuretic peptide) and a twofold increase in the risk of subsequent myocardial infarction. $^{27} 28 \beta$-Blockers may ameliorate these effects and result in improved survival in the peri-exacerbation period and long term. ${ }^{10} 15$

Our observational study cannot be used to conclude that $\beta$-blockers are beneficial in COPD nor to determine the mechanisms underlying their potential protective effects though both cardiac and non-cardiac benefits can be postulated. While $\beta$-blockers have a clear cardioprotective effect and a proportion of exacerbations may have cardiovascular causes, $\beta$-blockers may also have beneficial effects against episodes driven primarily by respiratory pathology. Long-term administration of $\beta$-blockers in murine models resulted in a sevenfold to eightfold increase in pulmonary $\beta$-adrenoreceptor density, ${ }^{29}$ and this overexpression of $\beta$ receptors can in turn decrease bronchoconstriction. ${ }^{30}$ Chronic $\beta$-blocker administration can also reduce airway inflammation and decrease mucus production. ${ }^{31}$ In addition, some selective $\beta$-blockers might modulate nitric oxide release, resulting in vasodilation and improved pulmonary haemodynamics, an effect that might also result from improvement in cardiac function with $\beta$-receptor blockade. ${ }^{32} \beta$-Blockers also reduce resting tachycardia, an independent predictor of mortality in COPD ${ }^{33}$ and might alleviate arrhythmias that can potentially cause cardiac and respiratory decompensation. ${ }^{34}$ They may also function by decreasing arrhythmogenesis and myocardial ischaemia associated with $\beta$ agonists that are used with greater frequency during the peri-exacerbation period. ${ }^{35}$

Previous retrospective studies support our findings; ${ }^{10}$ 12-14 3637 however, we add to the literature by using prospectively collected data from a well-characterised cohort with lung function measurements and spirometrically proven COPD. Building enthusiasm for $\beta$-blocker use was dampened by the findings of Ekstrom et al, ${ }^{18}$ who found in a prospective observational study that $\beta$-blocker therapy increased mortality in patients with severe COPD and on home oxygen. ${ }^{101213}$ We did not find such an effect on mortality, and in fact found that the significant effect size for exacerbation reduction persisted in this group. The potential for a major cardioprotective effect of $\beta$-blockers in this population is plausible as chronic hypoxia is a known cardiovascular risk factor and can lead to autonomic dysfunction and arrhythmias. ${ }^{38}$ The absence of a difference in mortality between patients taking $\beta$-blockers and those that were not could be because the study was underpowered to detect a difference but also supports the possibility that their apparent benefits on exacerbations were not solely due to a 'healthy' user phenomenon though such bias cannot be excluded in our observational study. Pertinent differences between our study and that reported by Ekstrom include that we adjusted for a number of additional measures of disease expression including symptoms, $\mathrm{FEV}_{1}$ and \%emphysema, which could be important as the use of home oxygen does not necessarily capture all aspects of disease severity. Subjects in Ekstrom's study were also a decade older (75.6 vs 65.5 years) but had better lung function (\%predicted $\mathrm{FEV}_{1} 40.5 \%$ vs $29.8 \%$ ) than our population with very severe disease. We speculate that the harmful effects of $\beta$-blockers in Ekstrom's report could be partly due to age-related effects and not due to COPD. Indeed, studies suggest that $\beta$-blockers might worsen composite cardiovascular outcomes in the elderly. ${ }^{39}$ Finally, Ekstrom's study did not assess the impact of $\beta$-blockers on exacerbations. We also found that the association between $\beta$-blockers and a reduction in exacerbations was greatest in patients with GOLD stage $\mathrm{B}$, a group in which cardiovascular mortality is the highest. ${ }^{40}$ Of note, there was no benefit associated with nonselective $\beta$-blockers.

In addition to physician-diagnosed CAD, we used CAC as another objective indicator of presence of atherosclerosis and $\mathrm{CAD}$, and its severity. Interestingly, the protective association of $\beta$-blockers was greater in those with CAC $<400$ than in those with CAC $>400$ in our cohort though this could be due to the low number of respiratory events in this smaller group of subjects with CAC $>400$. Alternatively, there are also data that greater calcium density is associated with lower risk of acute coronary events as these lesions may be more stable than less dense deposits and this phenomenon may extend to exacerbations triggered or worsened by coronary disease. $^{41} 42$ 
We also found that the use of CCBs was associated with an increased risk of severe exacerbations (though not a shorter time to first severe event or an association with total exacerbations). This was an unexpected result given that CCBs have been associated with potential benefits in asthma, ${ }^{43}$ but there are little published data to support either a harmful or beneficial effect of CCBs in COPD. Perhaps the best study examining CCB use in COPD did suggest that those patients taking the drugs had a higher risk of death and exacerbations than those taking $\beta$-blockers, consistent with our findings. ${ }^{9}$ No other study has directly compared exacerbation risk in those taking and not taking CCBs and such studies should be done.

Major strengths of the study are that the data were prospectively collected from a well-characterised COPD cohort with CT measures of emphysema and measurement of respiratory morbidity, and unlike a number of previous studies examining this issue, spirometry proven disease. Our study has several limitations. First, medication was self-reported; however, where possible, we confirmed drug prescriptions and dosage by examining medication containers. Second, patients with cardiac disease are more likely, and those with severe lung disease or greater dyspnoea less likely, to receive $\beta$-blockers, and though we performed propensity analyses to adjust for these factors, propensity analysis may not capture many intangible factors and residual bias remains possible. Third, we used the time to the first contact at which an exacerbation was reported rather than the exact date of the first exacerbation as the latter was not collected. Finally, mortality assessment was based on vital status information from the clinical centres; central adjudication has not yet been completed and thus cause-specific mortality has not been determined.

In summary, $\beta$-blocker use is associated with a reduction in COPD exacerbations, an effect not seen with other common cardiac medications. The findings of this study should be tested in a randomised, placebo-controlled study.

\section{Author affiliations}

${ }^{1}$ Division of Pulmonary, Allergy and Critical Care Medicine, UAB Lung Health Center, University of Alabama at Birmingham, Birmingham, Alabama, USA

${ }^{2}$ Department of Epidemiology, University of Colorado Anschutz Medical Campus, Aurora, Colorado, USA

${ }^{3}$ Division of Pulmonary and Critical Care Medicine, Brigham and Women's Hospital, Harvard Medical School, Boston, Massachusetts, USA

${ }^{4}$ Los Angeles Biomedical Research Institute at Harbor-UCLA Medical Center,

Torrance, California, USA

${ }^{5}$ Department of Preventive Medicine, University of Alabama at Birmingham, Birmingham, Alabama, USA

${ }^{6}$ Department of Radiology, University of Alabama at Birmingham, Birmingham, Alabama, USA

${ }^{7}$ Channing Division of Network Medicine, Brigham and Women's Hospital, Harvard Medical School, Boston, Massachusetts, USA

${ }^{8}$ Division of Pulmonary and Critical Care, National Jewish Health, Denver, Colorado, USA

${ }^{9}$ Birmingham VA Medical Center, Birmingham, Alabama, USA

Collaborators COPDGene Investigators.

Contributors Study design: SPB and MTD. Statistical analyses: SPB, MTD and YK. Data interpretation: SPB, JMW, GLK, GRW, MB, WCB, YK, HN, JEH, EKS, JC and MTD. Manuscript writing: SPB and MTD. Critical review of the manuscript for important intellectual content: SPB, JMW, GLK, GRW, MB, WCB, YK, HN, JEH, EKS, $J C$ and MTD.

Funding COPDGene is supported by NIH grant numbers R01 HL089897, R01 HL089856 and R01 HL122438. The coronary calcification data is supported by TRDRP Award No. 20XT-0014.

Competing Interests SPB has grant support from the American Heart Association. JMW's institution has contracts with GSK and Forest. JEH receives grant support from the National Health Lung and Blood Institute and from the American Diabetes Association. In the past three years, EKS received honoraria and consulting fees from Merck and grant support and consulting fees from GSK. GRW reports receiving consulting fees from Merck and GSK. WCB has received personal fees from DLA Piper. MTD has served as a consultant for GSK, BI, Boston Scientific and Ikaria. His institution has received research grant support from AHA, NHLBI, GSK and Forest and has received contracted support for enrolment in clinical trials from Aeris, AstraZeneca, BI, Boston Scientific, Centocor, GSK, Forest, Otsuka, Pearl, Pfizer, PneumRx and Pulmonx.

Patient consent All participants provided written informed consent prior to study enrolment. Follow-up data on exacerbations were prospectively obtained by contacting subjects every 3-6 months through an automated telephony system, by internet data collection or by research coordinators using a validated questionnaire.

Ethics approval The institutional review boards of all 21 participating centres approved the COPDGene study.

Provenance and peer review Not commissioned; externally peer reviewed.

\section{REFERENCES}

1 Vestbo J, Hurd SS, Agusti AG, et al. Global strategy for the diagnosis, management, and prevention of chronic obstructive pulmonary disease: GOLD executive summary. Am J Respir Crit Care Med 2013;187:347-65.

2 Puhan MA, Bachmann LM, Kleijnen J, et al. Inhaled drugs to reduce exacerbations in patients with chronic obstructive pulmonary disease: a network meta-analysis. BMC Med 2009;7:2

3 Bhatt SP, Dransfield MT. Chronic obstructive pulmonary disease and cardiovascular disease. Trans/ Res 2013:162:237-51.

4 van der Woude HJ, Zaagsma J, Postma DS, et al. Detrimental effects of beta-blockers in COPD: a concern for nonselective beta-blockers. Chest 2005;127:818-24.

5 Egred M, Shaw S, Mohammad B, et al. Under-use of beta-blockers in patients with ischaemic heart disease and concomitant chronic obstructive pulmonary disease. QJM 2005;98:493-7.

6 Salpeter S, Ormiston T, Salpeter E. Cardioselective beta-blockers for chronic obstructive pulmonary disease. Cochrane Database Syst Rev 2005;(4):CD003566.

7 Gottlieb SS, McCarter RJ, Vogel RA. Effect of beta-blockade on mortality among high-risk and low-risk patients after myocardial infarction. N Engl J Med 1998:339:489-97.

8 Chen J, Radford MJ, Wang Y, et al. Effectiveness of beta-blocker therapy after acute myocardial infarction in elderly patients with chronic obstructive pulmonary disease or asthma. J Am Coll Cardiol 2001;37:1950-6.

$9 \mathrm{Au} \mathrm{DH}$, Bryson CL, Fan VS, et al. Beta-blockers as single-agent therapy for hypertension and the risk of mortality among patients with chronic obstructive pulmonary disease. Am J Med 2004;117:925-31.

10 Rutten FH, Zuithoff NP, Hak E, et al. Beta-blockers may reduce mortality and risk of exacerbations in patients with chronic obstructive pulmonary disease. Arch Intern Med 2010;170:880-7.

11 van Gestel YR, Hoeks SE, Sin DD, et al. Impact of cardioselective beta-blockers on mortality in patients with chronic obstructive pulmonary disease and atherosclerosis. Am J Respir Crit Care Med 2008;178:695-700.

12 Short PM, Lipworth SI, Elder DH, et al. Effect of beta blockers in treatment of chronic obstructive pulmonary disease: a retrospective cohort study. BMJ 2011;342: d2549.

13 Farland MZ, Peters CJ, Williams JD, et al. beta-Blocker use and incidence of chronic obstructive pulmonary disease exacerbations. Ann Pharmacother 2013;47:651-6.

14 Brooks TW, Creekmore FM, Young DC, et al. Rates of hospitalizations and emergency department visits in patients with asthma and chronic obstructive pulmonary disease taking beta-blockers. Pharmacotherapy 2007;27:684-90.

15 Dransfield MT, Rowe SM, Johnson JE, et al. Use of beta blockers and the risk of death in hospitalised patients with acute exacerbations of COPD. Thorax 2008:63:301-5

16 Stefan MS, Rothberg MB, Priya A, et al. Association between beta-blocker therapy and outcomes in patients hospitalised with acute exacerbations of chronic obstructive lung disease with underlying ischaemic heart disease, heart failure or hypertension. Thorax 2012;67:977-84

17 Anderson JL, Adams CD, Antman EM, et al. 2012 ACCF/AHA focused update incorporated into the ACCF/AHA 2007 guidelines for the management of patients with unstable angina/non-ST-elevation myocardial infarction: a report of the American College of Cardiology Foundation/American Heart Association Task Force on Practice Guidelines. J Am Coll Cardiol 2013:61:e179-347.

18 Ekstrom MP, Hermansson AB, Strom KE. Effects of cardiovascular drugs on mortality in severe chronic obstructive pulmonary disease. Am J Respir Crit Care Med 2013;187:715-20.

19 Regan EA, Hokanson JE, Murphy JR, et al. Genetic epidemiology of COPD (COPDGene) study design. COPD 2010;7:32-43.

20 Han MK, Muellerova H, Curran-Everett D, et al. GOLD 2011 disease severity classification in COPDGene: a prospective cohort study. Lancet Respir Med 2013:1:43-50.

21 Budoff MJ, Nasir K, Kinney GL, et al. Coronary artery and thoracic calcium on noncontrast thoracic CT scans: comparison of ungated and gated examinations in 
patients from the COPD Gene cohort. J Cardiovasc Comput Tomogr 2011:5:113-18.

22 Wells JM, Washko GR, Han MK, et al. Pulmonary arterial enlargement and acute exacerbations of COPD. N Engl J Med 2012;367:913-21.

23 Han MK, Kazerooni EA, Lynch DA, et al. Chronic obstructive pulmonary disease exacerbations in the COPDGene study: associated radiologic phenotypes. Radiology 2011;261:274-82.

24 Williams MC, Murchison JT, Edwards LD, et al. Coronary artery calcification is increased in patients with COPD and associated with increased morbidity and mortality. Thorax 2014;69:718-23.

25 Austin PC. An introduction to propensity score methods for reducing the effects of confounding in observational studies. Multivariate Behav Res 2011;46:399-424.

26 Sin DD, Man SF. Chronic obstructive pulmonary disease as a risk factor for cardiovascular morbidity and mortality. Proc Am Thorac Soc 2005;2:8-11.

27 Donaldson GC, Hurst JR, Smith CJ, et al. Increased risk of myocardial infarction and stroke following exacerbation of COPD. Chest 2010;137:1091-7.

28 Chang $\mathrm{CL}$, Robinson SC, Mills GD, et al. Biochemical markers of cardiac dysfunction predict mortality in acute exacerbations of COPD. Thorax 2011;66:764-8.

29 Callaerts-Vegh Z, Evans KL, Dudekula N, et al. Effects of acute and chronic administration of beta-adrenoceptor ligands on airway function in a murine model of asthma. Proc Natl Acad Sci USA 2004;101:4948-53.

30 McGraw DW, Forbes SL, Mak JC, et al. Transgenic overexpression of beta (2)-adrenergic receptors in airway epithelial cells decreases bronchoconstriction. Am J Physiol Lung Cell Mol Physiol 2000;279:L379-89.

31 Nguyen LP, Omoluabi O, Parra S, et al. Chronic exposure to beta-blockers attenuates inflammation and mucin content in a murine asthma model. Am J Respir Cell Mol Biol 2008;38:256-62.

32 Dal Negro R. Pulmonary effects of nebivolol. Ther Adv Cardiovasc Dis 2009;3:329-34.
33 Nodari S, Metra M, Dei Cas L. Beta-blocker treatment of patients with diastolic heart failure and arterial hypertension. A prospective, randomized, comparison of the long-term effects of atenolol vs. nebivolol. Eur J Heart Fail 2003;5:621-7.

34 Bhatt SP, Nanda S, Kintzer JS. Arrhythmias as trigger for acute exacerbations of chronic obstructive pulmonary disease. Respir Med 2012;106:1134-8.

$35 \mathrm{Au} \mathrm{DH}$, Curtis JR, Every NR, et al. Association between inhaled beta-agonists and the risk of unstable angina and myocardial infarction. Chest 2002;121:846-51.

36 Du Q, Sun Y, Ding N, et al. Beta-blockers reduced the risk of mortality and exacerbation in patients with COPD: a meta-analysis of observational studies. PLOS ONE 2014;9:e113048.

37 Puente-Maestu L, Calle M, Ortega-Gonzalez A, et al. Multicentric study on the beta-blocker use and relation with exacerbations in COPD. Respir Med 2014;108:737-44.

38 Theofilogiannakos EK, Anogeianaki A, Tsekoura P, et al. Arrhythmogenesis in patients with stable chronic obstructive pulmonary disease. J Cardiovasc Med (Hagerstown) 2008;9:89-93.

39 Khan N, McAlister FA. Re-examining the efficacy of beta-blockers for the treatment of hypertension: a meta-analysis. CMAJ 2006;174:1737-42.

40 Lange $\mathrm{P}$, Marott JL, Vestbo J, et al. Prediction of the clinical course of chronic obstructive pulmonary disease, using the new GOLD classification: a study of the general population. Am J Respir Crit Care Med 2012;186:975-81.

41 Criqui $\mathrm{MH}$, Denenberg JO, Ix JH, et al. Calcium density of coronary artery plaque and risk of incident cardiovascular events. JAMA 2014;311:271-8.

42 Hou ZH, Lu B, Gao Y, et al. Prognostic value of coronary CT angiography and calcium score for major adverse cardiac events in outpatients. JACC CardiovasC Imaging 2012;5:990-9.

43 Girodet PO, Dournes G, Thumerel M, et al. Calcium channel blocker reduces airway remodeling in severe asthma. A proof-of-concept study. Am J Respir Crit Care Med 2015;191:876-83. 